Zürich University Preprint

ZU-TH 13/94, May 1994

hep-th/9406017

\title{
Einstein-Yang-Mills sphalerons and level crossing
}

\author{
Mikhail S. Volkov凹 \\ Institut für Theoretische Physik der Universität Zürich-Irchel, \\ Winterthurerstrasse 190, CH-8057 Zürich, \\ Switzerland \\ e-mail: volkov@physik.unizh.ch
}

\begin{abstract}
The fermion energy spectrum along paths which connect topologically distinct vacua in the Einstein-Yang-Mills theory passing through the gravitational sphaleron equilibrium solutions is investigated.
\end{abstract}

\footnotetext{
${ }^{1}$ On leave from Physical-Technical Institute of the Academy of Sciences of Russia, Kazan 420029, Russia
} 


\section{Introduction}

As is known, fermion number may be non-conserved in the standard model at high energies, in non-perturbative processes mediated by sphalerons [1]. Such processes correspond to overbarrier transitions between topologically distinct vacuum sectors of the theory, where the barrier height is given by the sphaleron mass. As the Chern-Simons (CS) number of the gauge field varies, this causes the anomalous nonconservation of fermion number.

In the first quantization picture, the change of fermion number is a result of level crossing [2]. Consider an adiabatic process, in which the boson fields interpolate between distinct vacuum sectors leading to a change of the CS number $N_{C S}$. The energy spectrum of massless fermions interacting with the boson background then depends parametrically on $N_{C S}$. When $N_{C S}$ varies, some of the energy levels cross zero ending up with an opposite sign of their energy. If the initial state corresponds to the filled Dirac sea, then (anti) fermions will be present in the final state. For the processes involving electroweak sphalerons, this picture was studied in Refs.[3], 近]. In addition, fermion production in dynamical decay of the sphaleron has been considered [5].

The aim of the present paper is to investigate the level crossing phenomenon arising within the context of the Einstein-Yang-Mills (EYM) theory. As is known, in this theory there exist regular particle-like solutions [6]. Such solutions relate to the critical points on the top of the potential barrier dividing distinct vacua in the theory, which allows them also to be interpreted as sphalerons [8]. One may expect that transition processes mediated by these EYM sphalerons become significant at high energies [9].

It has been shown recently that the EYM sphalerons possess zero energy fermion bound state [10]. The existence of this state indicates that some of the fermion energy levels cross zero when the evolving background field passes through the sphaleron field configuration. In the present paper we investigate the whole level crossing picture for the case when the background EYM fields interpolate between distinct vacua.

We define below paths which connect topologically distinct vacua in the EYM configuration space passing through the sphaleron equilibrium solutions. The field evolving adiabatically along such a path is used as the background field in the Dirac equation. To discretize the fermion energy spectrum, we compactify the 3-space by lifting the system onto a 3 -sphere with large but finite radius $R_{*}$, and thus the distance between energy levels is of the order of $1 / R_{*}$. For this geometrical regularization, we determine analytically the free fermion energy levels and zero fermion modes as well. In addition, we derive a simple analytic approximation for the whole level crossing spectrum, which gives qualitatively the right result. Finally, we solve the Dirac equation numerically and find the spectrum precisely. Our result is depicted in Fig.3 and shows explicitly the phenomenon of level crossing appearing in the EYM theory.

\section{EYM sphalerons and vacuum to vacuum paths}

Consider the action of the EYM theory for the $S U(2)$ gauge group

$$
S_{E Y M}=-\frac{1}{16 \pi G} \int R \sqrt{-g} d^{4} x-\frac{1}{2} \int \operatorname{tr} F_{\mu \nu} F^{\mu \nu} \sqrt{-g} d^{4} x .
$$


Here $F_{\mu \nu}=\partial_{\mu} A_{\nu}-\partial_{\nu} A_{\mu}-i e\left[A_{\mu}, A_{\nu}\right]$ is the matrix valued gauge field tensor, $e$ is the gauge coupling constant, $A_{\mu}=A_{\mu}^{a} \tau^{a} / 2$, and $\tau^{a}(a=1,2,3)$ are the Pauli matrices. Our sign conventions are those of Landau \& Lifshitz [20].

We choose the spherically symmetric gauge field as follows

$$
A=A_{\mu} d x^{\mu}=\frac{i}{2 e}(1-w) U d U^{-1}, \quad U=\exp \left(i \frac{\pi}{2} n^{a} \tau^{a}\right),
$$

where $n^{a}=(\sin \vartheta \cos \varphi, \sin \vartheta \sin \varphi, \cos \vartheta)$, and the gravitational field is parameterized by

$$
d s^{2}=l_{e}^{2}\left(\left(1-\frac{2 m}{r}\right) \sigma^{2} d t^{2}-\frac{d r^{2}}{1-2 m / r}-r^{2}\left(d \vartheta^{2}+\sin ^{2} \vartheta d \varphi^{2}\right)\right) .
$$

Here $l_{e}=\sqrt{4 \pi} l_{p l} / e$ is the only dimensional quantity in the problem ( $l_{p l}$ being Planck's length); the functions $w, m$ and $\sigma$ depend on $t$ and $r$.

For the static case, the corresponding Lagrangian equations read

$$
\begin{gathered}
\left(\left(1-\frac{2 m}{r}\right) \sigma w^{\prime}\right)^{\prime}=\sigma \frac{w\left(w^{2}-1\right)}{r^{2}}, \\
m^{\prime}=\left(1-\frac{2 m}{r}\right) w^{\prime 2}+\frac{\left(w^{2}-1\right)^{2}}{2 r^{2}}, \quad \sigma^{\prime}=2 \frac{w^{\prime 2}}{r} \sigma .
\end{gathered}
$$

These equations are known to possess regular, asymptotically flat solutions discovered by Bartnik and McKinnon [6]. These BK solutions are labeled by an integer, $n$. The function $w(r)$ for the $n$-th solution, $w_{n}(r)$, has $n$ nodes in the domain $0<r<\infty$ and satisfies the following boundary conditions:

$$
w_{n}(r)=1+O\left(r^{2}\right) \text { as } r \rightarrow 0, \quad w_{n}(r)=(-1)^{n}+\alpha_{n} / r+O\left(1 / r^{2}\right) \quad \text { as } r \rightarrow \infty,
$$

where $\alpha_{n}$ are numerically known constants [7]. The corresponding metric functions $m_{n}(r)$ and $\sigma_{n}(r)$ increase monotonically from $m_{n}(0)=0$ to $m_{n}(\infty)=m_{n}$ and from $\sigma_{n}(0)=\sigma_{n}$ to $\sigma_{n}(\infty)=1$, respectively (see Fig.1). The ADM masses of these solutions are $M_{n}=\sqrt{4 \pi} M_{p l} m_{n} / e$, with $M_{p l}$ being Planck's mass, where $m_{n}$ increases as $n$ grows from the minimal value $m_{1}=0.828$ to $m_{\infty}=1$.

As was shown in Ref. [8], the odd- $n$ BK solutions may be treated as sphalerons. This interpretation is based on the fact that, through any such solution, one may find a one-parameter family of fields which interpolates between topologically distinct EYM vacua. If $\lambda$ denotes the parameter of the family, the corresponding gauge field can be chosen as follows [8]

$$
A_{\mu} d x^{\mu}=\frac{i}{2 e}\left(1-w_{n}(r)\right) U d U^{-1}, \quad U=\exp \left(i \lambda n^{a} \tau^{a}\right)
$$

where $\lambda$ ranges from zero to $\pi$. The metric functions $m$ and $\sigma$ in (3) are given by

$$
\begin{gathered}
\sigma(r)=\exp \left\{-2 \sin ^{2} \lambda \int_{r}^{\infty} w_{n}^{\prime 2} \frac{d r}{r}\right\}, \\
m(r)=\frac{\sin ^{2} \lambda}{\sigma(r)} \int_{0}^{r}\left(w_{n}^{\prime 2}+\sin ^{2} \lambda \frac{\left(w_{n}^{2}-1\right)^{2}}{2 r^{2}}\right) \sigma d r .
\end{gathered}
$$

It is worth noting that, for any value of $\lambda$, the fields of this family satisfy the two Einstein equations $G_{0}^{0}=8 \pi G T_{0}^{0}$ and $G_{r}^{r}=8 \pi G T_{r}^{r}$; when $\lambda=\pi / 2$ these equations reduce to those 
in (5) [8]. Obviously, for $\lambda=\pi / 2$, the fields (7),(8) coincide with the $n$-th BK solution field. When $\lambda$ approaches zero or $\pi$ values, the spacetime metric becomes flat and the gauge field (7) vanishes. Thus, the family of fields (7),(8) describes a loop (in the EYM configuration function space) which interpolates between the trivial vacuum and the $n$-th BK solution when $\lambda$ runs from 0 to $\pi / 2$, and returns back to the vacuum as $\lambda$ changes from $\pi / 2$ to $\pi$.

It is important that this loop can be transformed to a path interpolating between neighbouring EYM vacua with different winding numbers of the gauge field. The gauge transformation

$$
A_{\mu} \rightarrow U\left(A_{\mu}+\frac{i}{e} \partial_{\mu}\right) U^{-1}, \text { with } U=\exp \left(i \frac{\lambda}{2}\left(w_{n}-1\right) n^{a} \tau^{a}\right)
$$

yields

$$
\begin{gathered}
A_{\mu} d x^{\mu}=\frac{i}{2 e}\left(1-w_{n}(r)\right) U_{+} d U_{+}^{-1}+\frac{i}{2 e}\left(1+w_{n}(r)\right) U_{-} d U_{-}^{-1}, \\
U_{ \pm}=\exp \left(i \lambda\left(w_{n} \pm 1\right) n^{a} \tau^{a} / 2\right)
\end{gathered}
$$

whereas the gauge invariant metric functions (8) remain unchanged. One can see that, when $\lambda$ runs from zero to $\pi$, the field (10) interpolates between two different pure gauges. We will refer to the gauge field potentials (17) and (10) as gauge field represented in the gauge $I$ and in the gauge $I I$, respectively. Notice that, in the gauge $I I$, one has $A_{a}=O(r)$ when $r \rightarrow 0$ and $A_{a}=O\left(1 / r^{2}\right)$ as $r \rightarrow \infty$, with $A_{a}$ being the field component with respect to an orthonormal frame.

Let $\lambda$ depend adiabatically on time, in such a way that $\lambda(t=-\infty)=0$ and $\lambda(t=\infty)=\pi$. Then, in the gauge $I$, the time component of the field appears, whereas in the gauge $I I$ one has $A_{0}=0$. Recall the Chern-Simons (CS) current and its divergence

$$
K^{\mu}=\frac{e^{2}}{8 \pi^{2}} \operatorname{tr} \frac{\varepsilon^{\mu \nu \alpha \beta}}{\sqrt{-g}} A_{\nu}\left(\nabla_{\alpha} A_{\beta}-\frac{2 i e}{3} A_{\alpha} A_{\beta}\right), \quad \nabla_{\alpha} K^{\alpha}=\frac{e^{2}}{16 \pi^{2}} \operatorname{tr} F_{\mu \nu} \tilde{F}^{\mu \nu}
$$

where $\nabla_{\alpha}$ is the covariant derivative, and $\tilde{F}^{\mu \nu}$ is dual tensor. Integration leads to the following relation:

$$
\frac{e^{2}}{16 \pi^{2}} \int_{-\infty}^{t} d t \int d^{3} x \sqrt{-g} t r F_{\mu \nu} \tilde{F}^{\mu \nu}=\left.\int d^{3} x \sqrt{-g} K^{0}\right|_{-\infty} ^{t}+\int_{-\infty}^{t} d t \oint \vec{K} d \vec{\Sigma} .
$$

In the temporal gauge $I I$, the surface integral entering the right hand side vanishes, while the gauge invariant left hand side is 8

$$
\frac{3}{2 \pi} \int_{-\infty}^{t} d t \dot{\lambda} \sin ^{2} \lambda \int_{0}^{\infty} d r w_{n}^{\prime}\left(w_{n}^{2}-1\right)=\left.\frac{3}{4 \pi}(\lambda-\sin \lambda \cos \lambda)\left(\frac{1}{3} w_{n}^{3}-w_{n}\right)\right|_{w_{n}(0)=1} ^{w_{n}(\infty)=-1} .
$$

Noting that the gauge potential (10) vanishes at $\lambda=0$, one obtains the following value of the CS number along the path (10) [8], [9]:

$$
N_{C S}=\left.\int \sqrt{-g} K^{0} d^{3} x\right|_{t}=\frac{1}{\pi}(\lambda-\sin \lambda \cos \lambda),
$$

which changes from zero to one as $\lambda$ runs from 0 to $\pi$ (see also Ref.[11] for a quite different approach to the calculation of the CS numbers). 
Thus the fields (3), (8), (10) interpolate between two EYM vacua with different winding numbers of the gauge field, and coincide with the (gauge transformed) field of the $n$-th BK solution when $\lambda=\pi / 2$. This allows one to treat odd- $n$ BK solutions as sphalerons, "lying" on the top of the potential barrier separating distinct topological vacuum sectors of the theory. The profile of this barrier may be obtained from Eq.(8) because the metric function $m(r)$ obeys the initial value constraint, which allows us to define the ADM energy [8]:

$$
\begin{gathered}
U(\lambda)=\lim _{r \rightarrow \infty} m(r)= \\
=\sin ^{2} \lambda \int_{0}^{\infty}\left(w_{n}^{\prime 2}+\sin ^{2} \lambda \frac{\left(w_{n}^{2}-1\right)^{2}}{2 r^{2}}\right) \exp \left(-2 \sin ^{2} \lambda \int_{r}^{\infty} w_{n}^{\prime 2} \frac{d r}{r}\right) d r,
\end{gathered}
$$

(a plot of the function $U\left(N_{C S}(\lambda)\right)$ for $n=1$ is depicted in Fig.2).

\section{Compactification of the 3-space}

Our aim is to investigate the energy spectrum of chiral fermions in the external fields (7), (3), (8) which form the potential barrier (15). As one expects, the change of the CS number across the barrier causes a non-trivial spectral flow. This concept is, however, not meaningful since the energy spectrum is purely continuous. Therefore, we apply some regularization procedure to discretize the spectrum. The most natural way is to compactify the 3 -space by lifting the system onto a 3 -sphere with large but finite radius $R_{*}$; the limit $R_{*} \rightarrow \infty$ being eventually implied (see also Ref. [4] for other choice of the regularization scheme).

For this we multiply the spatial part of the line element (3) by an appropriate conformal factor

$$
d s^{2}=l_{e}^{2}\left(\left(1-\frac{2 m}{r}\right) \sigma^{2} d t^{2}-\left(1+\frac{r^{2}}{4 R_{*}^{2}}\right)^{-2}\left\{\frac{d r^{2}}{1-2 m / r}+r^{2}\left(d \vartheta^{2}+\sin ^{2} \vartheta d \varphi^{2}\right)\right\}\right),
$$

with functions $m$ and $\sigma$ still given by Eqs.(8). When parameter $\lambda$ in (8) interpolates between zero and $\pi$ values, this line element describes an everywhere regular geometry on a manifold whose spatial section is topologically $S^{3}$. When $\lambda=0, \pi$, one has $m=$ $0, \sigma=1$, so that the spatial section is the 3 -sphere with radius $R_{*}$ and standard metric.

The lift of the gauge field is locally well defined by Eqs.(7) or (10). However, in the gauge $I$, the field is singular at $\tilde{r}=0$, where $\tilde{r}=1 / r$ (we will call this point north pole). Consider the gauge $I I$. In the vicinity of the north pole, the components of the field (10) with respect to an orthonormal frame are

$$
A_{a}=\frac{\alpha_{n}}{R_{*}^{2}} a_{a}(\vartheta, \varphi)+O(\tilde{r}),
$$

with $a_{a}$ being some functions of the angular variables, the constant $\alpha_{n}$ is the same as that entering Eq.(6). One can see that this field is not defined uniquely at $\tilde{r}=0$. One can not improve the situation just by performing a further gauge transformation in the vicinity of the pole (because the maximal decay rate of the field (10), $A_{a} \sim 1 / r^{2}$, is fixed by the asymptotical behaviour of the corresponding gauge invariant energy density: $\left.T_{0}^{0} \sim 1 / r^{6}\right)$. Notice, however, that for our geometrical regularization, the normalized fermion modes 
always vanish at the north pole (see Eq.(47)), so that this ambiguity does not enter the Dirac equation.

One can understand this also as follows. Let us introduce for a moment an additional regularization, demanding for the amplitude $w_{n}$, in the vicinity of the north pole, to tend faster to its asymptotical value:

$$
w_{n}(r)=(-1)^{n}+\alpha_{n} / r^{1+\epsilon}+O\left(1 / r^{2}\right), \quad \text { where } \epsilon \rightarrow+0 .
$$

The gauge field is then everywhere regular, whereas the field topology, that is, the CS number, remains the same. It turns out that, in the vicinity of the pole, the regular power series solution for fermions converges uniformly as $\epsilon \rightarrow 0$ to that corresponding to $\epsilon=0$. Thus fermions do not feel the $\epsilon$-dependence of the field.

We assume therefore that Eq.(10) specifies the regular gauge field on a compact manifold whose geometry is described by the line element (16).

\section{Fermions on an EYM background}

Our next task is to perform separation of variables in the Dirac equation. For the sake of generality, we will consider in this section massive doublet fermions interacting with arbitrary time dependent, spherically symmetric EYM background fields. Some particular cases of this problem were considered previously in Refs. 15], [10].

Consider the action of an $S U(2)$ doublet of fermions in an EYM background

$$
S_{f}=\int \bar{\Psi}\left(\gamma^{\mu}\left(i \nabla_{\mu}+e A_{\mu}\right)-M\right) \Psi \sqrt{-g} d^{4} x
$$

with $M$ being fermion mass; this action is invariant under the gauge transformations

$$
A_{\mu} \rightarrow U\left(A_{\mu}+\frac{i}{e} \partial_{\mu}\right) U^{-1}, \quad \Psi \rightarrow U \Psi, \quad U \in S U(2)
$$

It is most economic for our purposes to make use of the Newman-Penrose (NP) formalism of spin coefficients 12]. This formalism is known to be a convenient tool for studying the wave equations of fields of various spin in the algebraically distinguished spaces of general relativity [14], [15]. In the spherically symmetric case, the role of the spinor angular functions is played then by the spin-weighted spherical harmonics. (Notice that, in our problem, the separation of variables in terms of spherical spinors can also be performed [16], but the calculations are somewhat tedious.)

In spinor representation, bispinor $\Psi$ decomposes as $\Psi=\left(\begin{array}{c}\phi^{A} \\ \bar{\xi}_{A^{\prime}}\end{array}\right)$. In the chiral limit, two component spinors $\phi^{A}$ and $\bar{\xi}_{A^{\prime}}$ (they carry also isospin indices) become righthanded and left-handed parts of bispinor $\Psi$ respectively. The Dirac equation reads

$$
\left(\nabla_{A A^{\prime}}-i e A_{A A^{\prime}}\right) \phi^{A}=-i \frac{M}{\sqrt{2}} \bar{\xi}_{A^{\prime}}, \quad\left(\nabla^{A A^{\prime}}-i e A^{A A^{\prime}}\right) \bar{\xi}_{A^{\prime}}=-i \frac{M}{\sqrt{2}} \phi^{A}
$$

where the spin-tensors $\nabla_{A A^{\prime}}$ and $A_{A A^{\prime}}$ are related to the covariant derivative operator $\nabla_{\mu}$ and the gauge field potential $A_{\mu}$, via van der Waerden connection quantities $\sigma_{A A^{\prime}}^{\mu}$ |12):

$$
\nabla_{\mu} \leftrightarrow \nabla_{A A^{\prime}}=\sigma_{A A^{\prime}}^{\mu} \nabla_{\mu}, \quad A_{\mu} \leftrightarrow A_{A A^{\prime}}=\sigma_{A A^{\prime}}^{\mu} A_{\mu}
$$


The conserved current is

$$
J^{\mu} \leftrightarrow \phi_{1}^{A} \bar{\phi}_{2}^{A^{\prime}}+\xi_{1}^{A} \bar{\xi}_{2}^{A^{\prime}}
$$

which gives the inner product

$$
<\Psi_{2} \mid \Psi_{1}>=\int J^{0} \sqrt{-g} d^{3} x=\int \sigma_{A A^{\prime}}^{0}\left(\phi_{1}^{A} \bar{\phi}_{2}^{A^{\prime}}+\xi_{1}^{A} \bar{\xi}_{2}^{A^{\prime}}\right) \sqrt{-g} d^{3} x .
$$

Introduce a spinor dyad $\left(o^{A}, \iota^{A}\right), o_{A} \iota^{A}=1$, which defines a null NP tetrad $(l, n, m, \bar{m})$ according to the scheme

$$
l^{\mu} \leftrightarrow o^{A} \bar{o}^{A^{\prime}}, \quad n^{\mu} \leftrightarrow \iota^{A} \bar{\iota}^{A^{\prime}}, \quad m^{\mu} \leftrightarrow o^{A} \bar{\iota}^{A^{\prime}}, \quad \bar{m}^{\mu} \leftrightarrow \iota^{A} \bar{o}^{A^{\prime}},
$$

non-zero scalar products being $l^{\mu} n_{\mu}=-m^{\mu} \bar{m}_{\mu}=1$. Expanding $\phi^{A}$ and $\bar{\xi}_{A^{\prime}}$ over the spinor basis,

$$
\phi^{A}=\Phi_{0} o^{A}+\Phi_{1} \iota^{A}, \quad \bar{\xi}_{A^{\prime}}=\Xi_{0} \bar{o}_{A^{\prime}}+\Xi_{1} \bar{\iota}_{A^{\prime}},
$$

and using the NP quantities

$$
\bar{o}^{A^{\prime}} \nabla_{A A^{\prime}} O^{A}=\varepsilon-\rho, \quad \bar{o}^{A^{\prime}} \nabla_{A A^{\prime}} \iota^{A}=\pi-\alpha, \quad \bar{\iota}^{A^{\prime}} \nabla_{A A^{\prime}} O^{A}=\beta-\tau, \quad \iota^{A^{\prime}} \nabla_{A A^{\prime}} \iota^{A}=\mu-\gamma,
$$

one obtains the Dirac equation as

$$
\begin{gathered}
\left(D+\varepsilon-\rho-i e A_{l}\right) \Phi_{0}+\left(\bar{\delta}+\pi-\alpha-i e A_{\bar{m}}\right) \Phi_{1}=i \frac{M}{\sqrt{2}} \Xi_{1}, \\
\left(\delta+\beta-\tau-i e A_{m}\right) \Phi_{0}+\left(\Delta+\mu-\gamma-i e A_{n}\right) \Phi_{1}=-i \frac{M}{\sqrt{2}} \Xi_{0}, \\
\left(D+\varepsilon^{*}-\rho^{*}-i e A_{l}\right) \Xi_{0}+\left(\delta+\pi^{*}-\alpha^{*}-i e A_{m}\right) \Xi_{1}=-i \frac{M}{\sqrt{2}} \Phi_{1}, \\
\left(\bar{\delta}+\beta^{*}-\tau^{*}-i e A_{\bar{m}}\right) \Xi_{0}+\left(\Delta+\mu^{*}-\gamma^{*}-i e A_{n}\right) \Xi_{1}=i \frac{M}{\sqrt{2}} \Phi_{0} .
\end{gathered}
$$

Here the standard definitions used are: $D=l^{\mu} \partial_{\mu}, \Delta=n^{\mu} \partial_{\mu}, \delta=m^{\mu} \partial_{\mu}$, and also $A_{l}=l^{\mu} A_{\mu}, A_{n}=n^{\mu} A_{\mu}, A_{m}=m^{\mu} A_{\mu}, A_{\bar{m}}=\bar{m}^{\mu} A_{\mu}=A_{m}^{\dagger}$; asterisk denotes complex conjugation.

Let us choose the spacetime metric in the form

$$
d s^{2}=e^{2 a} d t^{2}-e^{2 b} d r^{2}-R^{2}\left(d \vartheta^{2}+\sin ^{2} \vartheta d \varphi^{2}\right),
$$

where functions $a, b$ and $R$ depend on $t$ and $r$. The general spherical gauge field [17] can be represented as follows:

$$
e A=W_{0} \hat{L}_{1} d t+W_{1} \hat{L}_{1} d r+\left\{p_{2} \hat{L}_{2}-\left(1-p_{1}\right) \hat{L}_{3}\right\} d \vartheta+\left\{\left(1-p_{1}\right) \hat{L}_{2}+p_{2} \hat{L}_{3}\right\} \sin \vartheta d \varphi
$$

where

$$
\hat{L}_{1}=\frac{1}{2} n^{a} \tau^{a}=\sin \vartheta \cos \varphi \hat{T}_{1}+\sin \vartheta \sin \varphi \hat{T}_{2}+\cos \vartheta \hat{T}_{3}, \hat{L}_{2}=\partial_{\vartheta} \hat{L}_{1}, \quad \hat{L}_{3}=\frac{1}{\sin \vartheta} \partial_{\varphi} \hat{L}_{1},
$$

with $\hat{T}_{a}=\tau^{a} / 2 ; W_{0}, W_{1}, p_{1}, p_{2}$ being functions of $t$ and $r$. The gauge transformation (20) generated by

$$
U=\exp \left(i \Omega(t, r) \hat{L}_{1}\right)
$$


preserves the form of the field (25), altering the functions $W_{0}, W_{1}, p_{1}, p_{2}$ as

$$
W_{0} \rightarrow W_{0}+\dot{\Omega}, \quad W_{1} \rightarrow W_{1}+\Omega^{\prime}, \quad p_{ \pm}=p_{1} \pm i p_{2} \rightarrow \exp ( \pm i \Omega) p_{ \pm} ;
$$

here dot and prime denote differentiation with respect to $t$ and $r$, respectively.

We choose the null NP tetrad as

$$
l^{\mu}=\left(e^{-a}, e^{-b}, 0,0\right), \quad n^{\mu}=\frac{1}{2}\left(e^{-a},-e^{-b}, 0,0\right), \quad m^{\mu}=\frac{1}{\sqrt{2} R}\left(0,0,1, \frac{i}{\sin \vartheta}\right),
$$

the non-vanishing NP coefficients being

$$
\begin{gathered}
\rho=-\frac{1}{R}\left(\dot{R} e^{-a}+R^{\prime} e^{-b}\right), \quad \mu=\frac{1}{2 R}\left(\dot{R} e^{-a}-R^{\prime} e^{-b}\right), \quad \gamma=-\frac{1}{4}\left(\dot{b} e^{-a}-a^{\prime} e^{-b}\right), \\
\varepsilon=\frac{1}{2}\left(\dot{b} e^{-a}+a^{\prime} e^{-b}\right), \quad \alpha=-\beta=-\frac{c t g \vartheta}{2 \sqrt{2} R} .
\end{gathered}
$$

The tetrad components of the gauge field potential are

$$
e A_{l}=\left(e^{-a} W_{0}+e^{-b} W_{1}\right) \hat{L}_{3}, e A_{n}=\frac{1}{2}\left(e^{-a} W_{0}-e^{-b} W_{1}\right) \hat{L}_{3}, i e A_{m}=\frac{1}{\sqrt{2} R}\left(p_{+}-1\right) \hat{L}_{+},
$$

where $\hat{L}_{ \pm}=\hat{L}_{1} \pm i \hat{L}_{2}$. Using Eqs.(30),(31), the Dirac equation (23) takes the form

$$
\begin{aligned}
& \hat{\mathcal{D}}_{+} \Phi_{0}+\frac{1}{\sqrt{2} R}\left(\hat{\Lambda}_{-}+\frac{1}{2} \operatorname{ctg} \vartheta+\left(p_{-}-1\right) \hat{L}_{-}\right) \Phi_{1}=i \frac{M}{\sqrt{2}} \Xi_{1}, \\
& \hat{\mathcal{D}}_{-} \Phi_{1}+\frac{\sqrt{2}}{R}\left(\hat{\Lambda}_{+}+\frac{1}{2} \operatorname{ctg} \vartheta-\left(p_{+}-1\right) \hat{L}_{+}\right) \Phi_{0}=-i \sqrt{2} M \Xi_{0}, \\
& \hat{\mathcal{D}}_{+} \Xi_{0}+\frac{1}{\sqrt{2} R}\left(\hat{\Lambda}_{+}+\frac{1}{2} \operatorname{ctg} \vartheta-\left(p_{+}-1\right) \hat{L}_{+}\right) \Xi_{1}=-i \frac{M}{\sqrt{2}} \Phi_{1}, \\
& \hat{\mathcal{D}}_{-} \Xi_{1}+\frac{\sqrt{2}}{R}\left(\hat{\Lambda}_{-}+\frac{1}{2} \operatorname{ctg} \vartheta+\left(p_{-}-1\right) \hat{L}_{-}\right) \Xi_{0}=i \sqrt{2} M \Phi_{0},
\end{aligned}
$$

where

$$
\begin{gathered}
\hat{\mathcal{D}}_{ \pm}=e^{-a}\left(\partial_{t}+\frac{\dot{b}}{2}+\frac{\dot{R}}{R}-i W_{0} \hat{L}_{1}\right) \pm e^{-b}\left(\partial_{r}+\frac{a^{\prime}}{2}+\frac{R^{\prime}}{R}-i W_{1} \hat{L}_{1}\right) \\
\hat{\Lambda}_{ \pm}=\partial_{\vartheta} \pm \frac{i}{\sin \varphi} \partial_{\varphi}
\end{gathered}
$$

Introduce a pair of isotopic spinors:

$$
\chi^{+}=\left(\begin{array}{c}
\cos \frac{\vartheta}{2} e^{-i \varphi / 2} \\
\sin \frac{\vartheta}{2} e^{i \varphi / 2}
\end{array}\right), \quad \chi^{-}=\left(\begin{array}{c}
-\sin \frac{\vartheta}{2} e^{-i \varphi / 2} \\
\cos \frac{\vartheta}{2} e^{i \varphi / 2}
\end{array}\right),
$$

with the following properties

$$
\begin{gathered}
\hat{L}_{1} \chi^{ \pm}= \pm \frac{1}{2} \chi^{ \pm}, \quad \hat{L}_{ \pm} \chi^{\mp}=\chi^{ \pm}, \quad \hat{L}_{ \pm} \chi^{ \pm}=0, \\
\hat{\Lambda}_{ \pm} \chi^{ \pm}=\frac{1}{2} \operatorname{ctg} \vartheta \chi^{ \pm}, \quad\left(\hat{\Lambda}_{ \pm}+\frac{1}{2} \operatorname{ctg} \vartheta\right) \chi^{\mp}=\mp \chi^{ \pm} .
\end{gathered}
$$


To decouple angular variables in Eqs.(32) we make the ansatz

$$
\begin{aligned}
& \Phi_{0}=\frac{1}{R} e^{-a / 2}\left(\chi^{+} F_{0}^{+}{ }_{-1} Y_{j m}+\chi^{-} F_{0}^{-}{ }_{0} Y_{j m}\right), \quad \Phi_{1}=\frac{\sqrt{2}}{R} e^{-a / 2}\left(\chi^{+} F_{1}^{+}{ }_{0} Y_{j m}+\chi^{-} F_{1}^{-}{ }_{1} Y_{j m}\right), \\
& \Xi_{0}=\frac{1}{R} e^{-a / 2}\left(\chi^{+} Q_{0}^{+}{ }_{0} Y_{j m}+\chi^{-} Q_{0}^{-}{ }_{1} Y_{j m}\right),
\end{aligned}
$$

where ${ }_{s} Y_{j m}(\vartheta, \varphi)$ are spin weighted spherical harmonics [13], and the functions $F_{\sigma}^{ \pm}, Q_{\sigma}^{ \pm}$ $(\sigma=0,1)$ depend on $t$ and $r$ alone. A gauge transformation of the form (20), (27) induces the replacement

$$
F_{\sigma}^{ \pm} \rightarrow \exp ( \pm i \Omega / 2) F_{\sigma}^{ \pm}, \quad Q_{\sigma}^{ \pm} \rightarrow \exp ( \pm i \Omega / 2) Q_{\sigma}^{ \pm}
$$

Taking into account Eqs.(35) and also the following standard relations for the functions ${ }_{s} Y_{j m}$ 13]

$$
\left(\partial_{\vartheta} \mp \frac{i}{\sin \vartheta} \partial_{\varphi} \pm s \operatorname{ctg} \vartheta\right)_{s} Y_{j m}= \pm \sqrt{(j \pm s)(j \mp s+1)}{ }_{s \mp 1} Y_{j m}
$$

one can see that the ansatz (36) solves Eqs.(32), provided that the following equations hold

$$
\begin{gathered}
\hat{D}_{+}^{*} F_{0}^{+}+\frac{\Lambda}{R} F_{1}^{+}=i M Q_{1}^{+}, \\
\hat{D}_{-} F_{1}^{-}-\frac{\Lambda}{R} F_{0}^{-}=-i M Q_{0}^{-}, \\
\hat{D}_{+} F_{0}^{-}+\frac{p_{-}}{R} F_{1}^{+}+\frac{\Lambda}{R} F_{1}^{-}=i M Q_{1}^{-}, \\
\hat{D}_{-}^{*} F_{1}^{+}-\frac{p_{+}}{R} F_{0}^{-}-\frac{\Lambda}{R} F_{0}^{+}=-i M Q_{0}^{+}, \\
\hat{D}_{+}^{*} Q_{0}^{+}-\frac{p_{+}}{R} Q_{1}^{-}-\frac{\Lambda}{R} Q_{1}^{+}=-i M F_{1}^{+}, \\
\hat{D}_{-} Q_{1}^{-}+\frac{p_{-}}{R} Q_{0}^{+}+\frac{\Lambda}{R} Q_{0}^{-}=i M F_{0}^{-}, \\
\hat{D}_{+} Q_{0}^{-}-\frac{\Lambda}{R} Q_{1}^{-}=-i M F_{1}^{-}, \\
\hat{D}_{-}^{*} Q_{1}^{+}+\frac{\Lambda}{R} Q_{0}^{+}=i M F_{0}^{+},
\end{gathered}
$$

where $\Lambda=\sqrt{j(j+1)}$, and

$$
\hat{D}_{ \pm}=e^{-a}\left(\partial_{t}+\frac{\dot{b}-\dot{a}}{2}+\frac{i}{2} W_{0}\right) \pm e^{-b}\left(\partial_{r}+\frac{i}{2} W_{1}\right) .
$$

For the $S$-modes, $j=0$, the spherical harmonics ${ }_{ \pm 1} Y_{j m}$ vanish, and the resulting equations reduce to the $F_{0}^{+}=F_{1}^{-}=Q_{1}^{+}=Q_{0}^{-}=\Lambda=0$ case. One can see that the transformations (28), (37) leave Eqs.(38) invariant. 


\section{$5 \quad$ Fermion energy spectrum}

For the background field quantities entering the system (38), we choose now (8),(10) and (16), describing EYM fields of a vacuum-to-vacuum path. We treat these fields, in the adiabatic approximation, as a sequence of instantaneously static configurations, with $\lambda$ being a parameter. Our aim is to study the energy spectrum of fermions as a function of $\lambda$.

In the chiral limit, the two chiralities decouple. In the following, we consider just the right-handed spinor component. We are interested in the $S$-wave sector dynamics, because, as we expect, it is in this sector where the level crossing phenomenon will occur. The following combinations are introduced for the fermions functions surviving in the case:

$$
F_{0}^{-}+F_{1}^{+}=i \sqrt{2} e^{-i \omega t} \psi^{+}(r), \quad F_{0}^{-}-F_{1}^{+}=\sqrt{2} e^{-i \omega t} \psi^{-}(r) .
$$

The non-trivial equations (38) take now the form

$$
\begin{gathered}
\left(\frac{d}{d r}+\frac{p_{1}}{N}\right) \psi^{+}+\left(-\frac{\omega r^{2}}{\sigma N^{2}}\left(1+\frac{r^{2}}{4 R_{*}^{2}}\right)^{-1}+\frac{1}{2} W_{1}+\frac{p_{2}}{N}\right) \psi^{-}=0, \\
\left(\frac{d}{d r}-\frac{p_{1}}{N}\right) \psi^{-}+\left(\frac{\omega r^{2}}{\sigma N^{2}}\left(1+\frac{r^{2}}{4 R_{*}^{2}}\right)^{-1}-\frac{1}{2} W_{1}+\frac{p_{2}}{N}\right) \psi^{+}=0,
\end{gathered}
$$

where $N=\sqrt{r^{2}-2 m r}$; the functions $m$ and $\sigma$ are given by (8). The quantities specifying the background gauge field (10) (taken in the regular gauge $I I$ ) read

$$
W_{1}=\lambda w_{n}^{\prime}, \quad p_{1}=\cos \lambda \cos w_{n} \lambda+w_{n} \sin \lambda \sin w_{n} \lambda, \quad p_{2}=\cos \lambda \sin w_{n} \lambda-w_{n} \sin \lambda \cos w_{n} \lambda .
$$

Due to (28), (37), these equations possess the following gauge symmetry

$$
\begin{gathered}
\psi^{+} \rightarrow \psi^{+} \cos \frac{\Omega}{2}-\psi^{-} \sin \frac{\Omega}{2}, \quad \psi^{-} \rightarrow \psi^{+} \sin \frac{\Omega}{2}+\psi^{-} \cos \frac{\Omega}{2}, \\
W_{1} \rightarrow W_{1}+\Omega^{\prime}, \quad p_{1} \rightarrow p_{1} \cos \Omega-p_{2} \sin \Omega, \quad p_{2} \rightarrow p_{1} \sin \Omega+p_{2} \cos \Omega,
\end{gathered}
$$

where $\Omega=\Omega(r)$. Sometimes it is convenient to utilize this symmetry in order to search for the solutions to Eqs.(40), working not in the regular gauge $I I$ for the background gauge field, but in the gauge $I$ instead. The corresponding field parameters are simpler in this case:

$$
W_{1}=0, \quad p_{1}=\cos ^{2} \lambda+w_{n} \sin ^{2} \lambda, \quad p_{2}=\left(1-w_{n}\right) \sin \lambda \cos \lambda .
$$

Once the solution is found, one has to switch back to the regular gauge II using the transformation (42), with the parameter

$$
\Omega=\lambda\left(w_{n}-1\right)
$$

The conserved inner product $(22)$ reads

$$
<\psi_{2} \mid \psi_{1}>=\int d r \frac{r^{2}}{\sigma N^{2}}\left(1+\frac{r^{2}}{4 R_{*}^{2}}\right)^{-1}\left(\psi_{2}^{+} \psi_{1}^{+}+\psi_{2}^{-} \psi_{1}^{-}\right)
$$


The formal series expansions around $r=0$ and $r=\infty$ give the following boundary conditions for the normalizable solutions to Eqs.(40), (41)

$$
\begin{gathered}
\psi^{+}=O\left(r^{2}\right), \quad \psi^{-}=O(r), \quad \text { as } r \rightarrow 0, \\
\psi^{+}=O\left(\frac{1}{r}\right), \quad \psi^{-}=O\left(\frac{R_{*}^{2}}{r^{2}}\right), \quad \text { as } r \rightarrow \infty .
\end{gathered}
$$

For each value of $\lambda$, the solutions with the asymptotics (46) and (47) have to be matched by adjusting the value of $\omega$. As a result, one obtains the energy spectrum, $\omega(\lambda)$.

Before passing to tackling the problem numerically, it is helpful to investigate some particular cases. Firstly, consider the beginning of the vacuum-to-vacuum path, $\lambda=0$. Then, both in the $I$ and $I I$ gauges, one has $p_{1}=1, p_{2}=W_{1}=0$, that is, the gauge field is zero; also $m=0, \sigma=1$. Eqs. (40) describe in the case free fermions on $R \times S^{3}$. Introducing a new radial coordinate $x \in[0, \pi]$ by $r=R_{*}\left(1+r^{2} / 4 R_{*}^{2}\right) \sin x$, one can represent Eqs.(40) as

$$
\left(\frac{d}{d x}+\frac{1}{\sin x}\right) \psi^{+}-\frac{\nu}{2} \psi^{-}=0, \quad\left(\frac{d}{d x}-\frac{1}{\sin x}\right) \psi^{+}+\frac{\nu}{2} \psi^{-}=0,
$$

where $\nu=2 \omega R_{*}$. The general solution of these equations reads

$$
\psi=\left(\begin{array}{c}
\psi^{+} \\
\psi^{-}
\end{array}\right)=A\left(\begin{array}{c}
\nu \cos (\nu x / 2+B)-\operatorname{ctg}(x / 2) \sin (\nu x / 2+B) \\
-\nu \sin (\nu x / 2+B)+t g(x / 2) \cos (\nu x / 2+B)
\end{array}\right),
$$

with $A$ and $B$ being integration constants; $\nu \neq \pm 1$. This solution vanishes at $x=0$ only if $B=0$. When $\nu$ is an odd integer, the solution vanishes also at $x=\pi$. The values $\nu= \pm 1$ correspond to the degenerate case. The solution then includes power terms, and it can not be regular both at $x=0$ and $x=\pi$. One obtains therefore the following spectrum of fermions (see also [10])

$$
\omega(\lambda=0)=\frac{\nu}{2 R_{*}}, \quad \nu= \pm 3, \pm 5 \ldots
$$

Consider now the end point of the vacuum-to-vacuum path, $\lambda=\pi$. To find the spectrum, we observe that, in the gauge $I$, the gauge field parameters (43) take the same values both at $\lambda=0$ and $\lambda=\pi$. This means that the solution (49) can be used also when $\lambda=\pi$, but only in the gauge $I$. To pass to the gauge $I I$, one just performs the transformation (44), so that the spectrum (50) remains the same.

We conclude therefore that, as $\lambda$ varies from zero to $\pi$, the spectrum (50) maps into itself. Certainly, this does not mean that each energy level maps into itself. The level number, $\nu$, may change. For instance, some of the levels may cross zero, ending up with an opposite sign of their energy as $\lambda \rightarrow \pi$. Observe that, in the gauge $I$, Eqs.(40) are manifestly invariant under

$$
\omega \rightarrow-\omega, \quad \lambda \rightarrow \pi-\lambda, \quad \psi^{+} \rightarrow-\psi^{+}, \quad \psi^{-} \rightarrow \psi^{-} .
$$

This means that normalizable solution with zero energy may appear only if $\lambda=\pi / 2$, and one of the functions $\psi^{ \pm}$vanishes. In the gauge $I$, the corresponding solution (first discovered in Ref. [10]) can be found from (40):

$$
\psi^{+}=0, \quad \psi^{-}=\exp \left(\int^{r} d r \frac{p_{1}}{N}\right) ;
$$


to pass to the regular gauge $I I$, one uses (42), (44).

Thus, we can see that the spectrum maps into itself as $\lambda$ runs from zero to $\pi$, and also some of the levels cross zero at $\lambda=\pi / 2$. To get an idea about the whole level crossing picture, we use the following simple analytic approximation which gives qualitatively the right result. Let us put in (40) for any $\lambda, m=0, \sigma=1$, that is, we neglect the change of the geometry along the vacuum-to-vacuum path. Also, we replace the function $w_{n}$ in (41), (43) by a step function $w$ obeying the same boundary conditions:

$$
w_{n}(r) \rightarrow w(r)=1, \quad r<r_{0} ; \quad w(r)=-1, \quad r>r_{0} .
$$

Notice that the field topology remains intact, as the Chern-Simons number still changes in accordance with Eq.(14), for this number depends only on the boundary conditions for $w_{n}$ (see Eq.(13)). The normalizable zero energy solution (52) also exists in this case.

To find the solution for the fermion wave function, we observe that, in the gauge $I$, the function $w$ describes a field which vanishes when $r<r_{0}$ and is a pure gauge when $r>r_{0}$ (see Eq.(7)). The resulting solution is therefore obtained by matching the free fermion modes in the domain $r<r_{0}$ and the gauge transformed free modes in the region $r>r_{0}$. Let us use the same independent variable $x=x(r)$ as in (48). When $x<x_{0}=x_{0}\left(r_{0}\right)$, the regular solution is given by Eq.(49) with $B=0$ and $A=1$. To obtain the solution in the region $x>x_{0}$, one takes the solution (49) with arbitrary $A$ and $B$, and performs the gauge rotation (42) with $\Omega=2 \lambda$ (to find $\Omega$ one compares (7), (53) and (27)). Matching these solutions at $x=x_{0}$ specifies the values of $A$ and $B$. Next, the solution in the region $x>x_{0}$ has to be rotated to the gauge $I I$ and required to vanish at $x=\pi$, which gives the quantization condition. It suffices to demand for only one component to vanish, say $\psi^{+}$, as the other will then vanish automatically, due to (47). In the limit $x_{0} \rightarrow 0$, the quantization condition takes the following simple form

$$
\operatorname{ctg} \lambda=\frac{2}{3} \nu x_{0}\left(1+O\left(x_{0}\right)\right)+\frac{1}{4} x_{0}^{2}\left(\nu^{2}-1\right)\left(1+O\left(x_{0}\right)\right) \operatorname{tg}\left(\nu \frac{\pi}{2}\right) .
$$

This equation gives the spectrum $\omega(\lambda)=\nu(\lambda) / 2 R_{*}$. When $\lambda=0, \pi$, ctg $\lambda$ diverges, which requires $\nu= \pm 3, \pm 5, \ldots$, in agreement with Eq.(50). As $\lambda$ runs from zero to $\pi$, ctg $\lambda$ decreases from $+\infty$ to $-\infty$, which forces $\nu(\lambda)$ to vary from $\nu(0) \neq 3$ to $\nu(\pi)=\nu(0)-2$, and from $\nu(0)=3$ to $\nu(\pi)=-3$.

We arrive, therefore, at the following level crossing picture for the right-handed chiral fermions. When the CS number varies from zero to one, each energy level shifts downwards replacing the preceding one with the lower energy. The lowest positive energy level crosses zero when $N_{C S}=1 / 2$ and becomes eventually the highest negative energy level. If the initial state corresponds to the fermion vacuum, then one (left-handed) antifermion appears in the final state.

Finally, we turn to the numerical analysis. We consider the vacuum-to-vacuum path (10), (16) passing through the (lifted) ground state EYM sphaleron field configuration. The compactification radius is chosen to be $R_{*}=10^{3}$ which is much larger than the sphaleron size (the latter is of the order of unity; see Fig.1). Numerical integration of the Dirac equation (40) with the boundary conditions (46), (47) gives the spectrum. The result is depicted in Fig. 3 and confirms the qualitative picture described above. For the paths passing through the higher $(n>1)$ EYM sphalerons, the level crossing picture is qualitatively the same. 


\section{Conclusion}

Our results show explicitly the anomalous non-conservation of fermion number in the transition processes mediated by the EYM sphalerons. It was argued recently that such processes can be significant at high energies due to interference effects [9]. One may expect then an efficient fermion number non-conservation in the high energy limit. To determine more precisely the production of fermions, by going beyond the adiabatic approximation, requires a numerical solution of the full time dependent problem [5], [18. Our results may be of use also for further investigation of the quantum correction to the classical energy of the sphaleron barrier, arising from the fermion see [4], [19].

\section{Acknowledgments}

I would like to thank Professor N.Straumann for a careful reading of the manuscript, and also Jutta Kunz, A.Losev and A.Wipf for discussions.

This work was supported by the Swiss National Science Foundation.

\section{References}

[1] F.R.Klinkhamer, N.S.Manton, Phys.Rev.D 30 (1984) 2212.

[2] C.G.Callan, R.Dashen, D.J.Gross, Phys.Rev.D 17 (1978) 2717;

J.Kiskis, Phys.Rev.D 18 (1978) 3690;

N.H.Christ, Phys.Rev.D 21 (1980) 1591;

V.A.Matveev et al., Usp.Fiz.Nauk 156 (1988) 253 [Sov.Phys.Usp. 31 (1988) 916].

[3] J.Boguta, J.Kunz, Phys.Lett.B 154 (1985) 407;

A.Ringwald, Phys.Lett.B 213 (1988) 61;

J.Kunz, Y.Brihaye, Phys.Lett.B 304 (1993) 141;

[4] D.I.Diakonov et al. Preprint RUB-TPII-62/93, hep-ph/9311374;

[5] M.Hellmund, J.Kripfganz, Nucl.Phys.B 373 (1991) 749;

W.N.Cottingham, N.Hasan, Nucl.Phys.B 392 (1993) 39.

[6] R.Bartnik, J.McKinnon, Phys.Rev.Lett. 61 (1988) 141.

[7] H.P.Kunzle, A.K.M.Masood-ul-Alam, J.Math.Phys. 31 (1990) 928.

[8] D.V.Gal’tsov, M.S.Volkov, Phys.Lett.B 273 (1991) 255;

D.Sudarsky, R.M.Wald, Phys.Rev.D 46 (1992) 1453.

[9] M.S.Volkov, Preprint ZU-TH 32/93, hep-th/9312005, to appear in Phys.Lett.B.

[10] G.W.Gibbons, A.R.Steif, Phys.Lett.B 314 (1993) 13; Phys.Lett.B 320 (1993) 245.

[11] O.Brodbeck, N.Straumann, Journ.Math.Phys. 35 (1994) 899. 
[12] E.Newman, R.Penrose, Journ.Math.Phys. 3 (1962) 566.

[13] E.Newman, R.Penrose, Journ.Math.Phys. 7 (1966) 863;

J.N.Goldberg et al. Journ.Math.Phys. 8 (1967) 2155.

[14] S.Chandrasekhar, The Mathematical Theory of Black Holes, Oxford University Press, 1983;

G.W.Gibbons, Phys.Rev.D 15 (1977) 3530;

D.V.Gal'tsov, Particles and Fields Near Black Holes (in Russian), Moscow University Press, 1986;

M.S.Volkov, D.V.Gal'tsov, Sov.Theor.Math.Phys. 80 (1989) 716.

[15] D.V.Gal'tsov, E.E.Ershov Sov.Journ.Nucl.Phys. 47 (1988) 355.

[16] I.Sachs, private communication.

[17] R.F.Dashen, B.Hasslasher, A.Neveu Phys.Rev.D 10 (1974) 4138;

E.Witten, Phys.Rev.Lett. 38 (1977) 121.

[18] Z.H.Zhou, N.Straumann, Nucl.Phys.B 360 (1991) 180.

[19] G.Nolte, J.Kunz, Phys.Rev.D 48 (1993) 5905.

[20] L.D.Landau \& E.M.Lifshitz, The classical theory of fields (Pergamon, Oxford, 1975).

\section{Figure captions}

Fig.1. Behaviour of the gauge field function, $w_{1}(r)$, the metric functions, $m_{1}(r), \sigma_{1}(r)$, and the radial energy density $r^{2} T_{0}^{0}$ for the ground state $(n=1)$ EYM sphaleron solution. In the units used, the sphaleron has the typical size of the order of unity.

Fig.2. The potential barrier dividing neighbouring topological EYM vacua. The top of the barrier is the position of the ground state EYM sphaleron.

Fig.3. The fermion energy spectrum along the path which interpolates between distinct topological EYM vacua passing through the ground state sphaleron field. 
This figure "fig1-1.png" is available in "png" format from: http://arxiv.org/ps/hep-th/9406017v1 


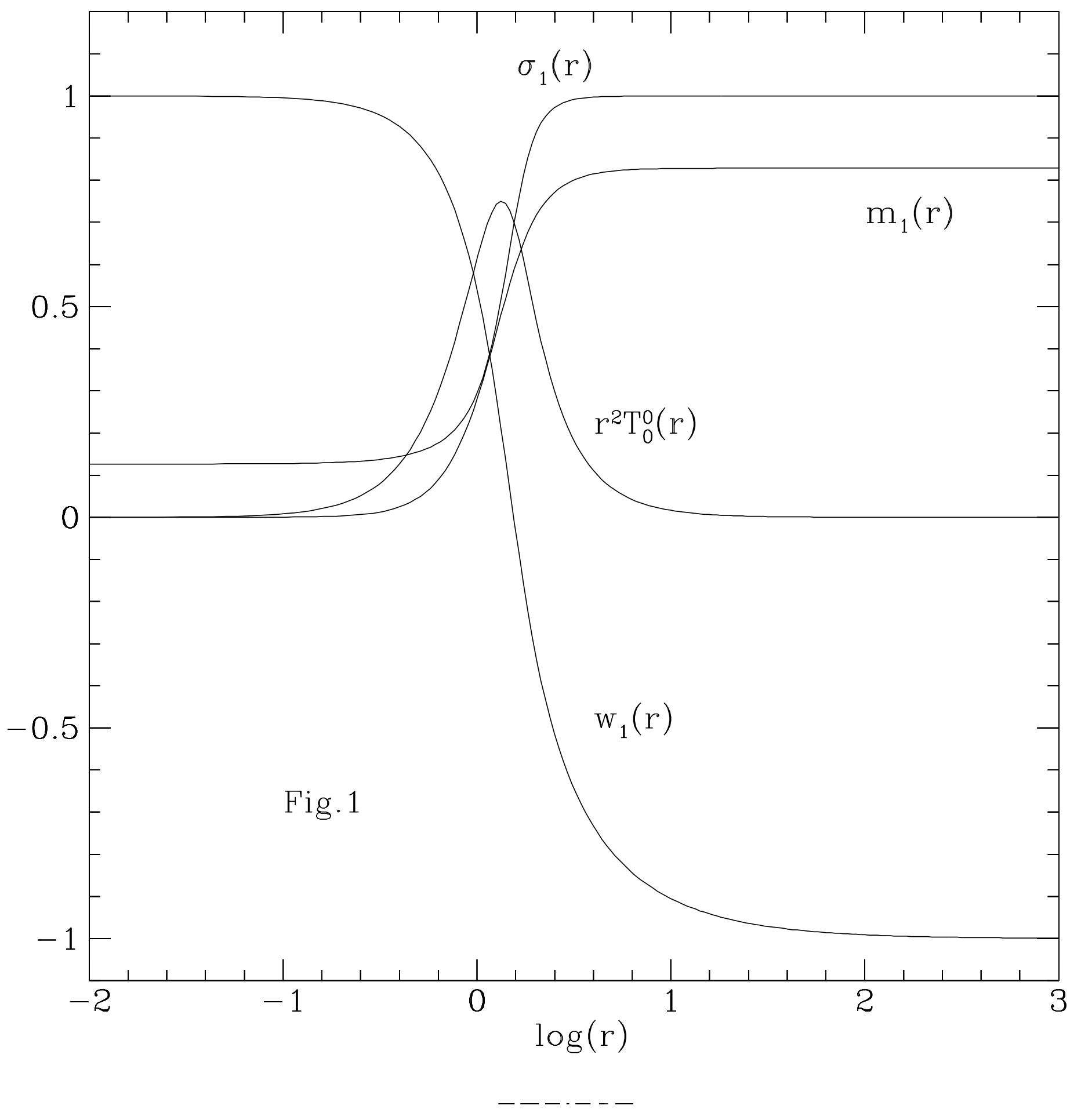


This figure "fig1-2.png" is available in "png" format from: http://arxiv.org/ps/hep-th/9406017v1 


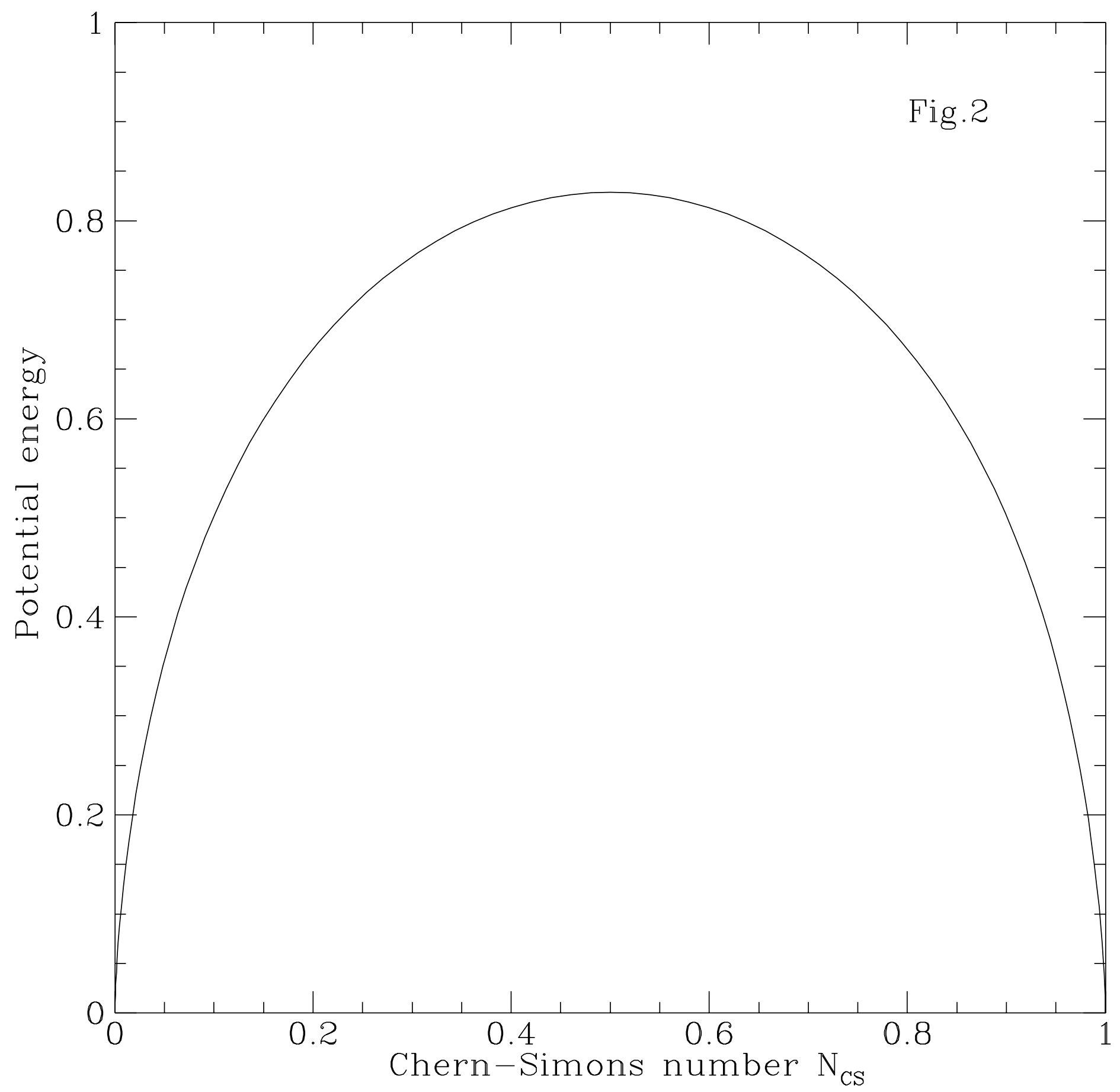


This figure "fig1-3.png" is available in "png" format from: http://arxiv.org/ps/hep-th/9406017v1 


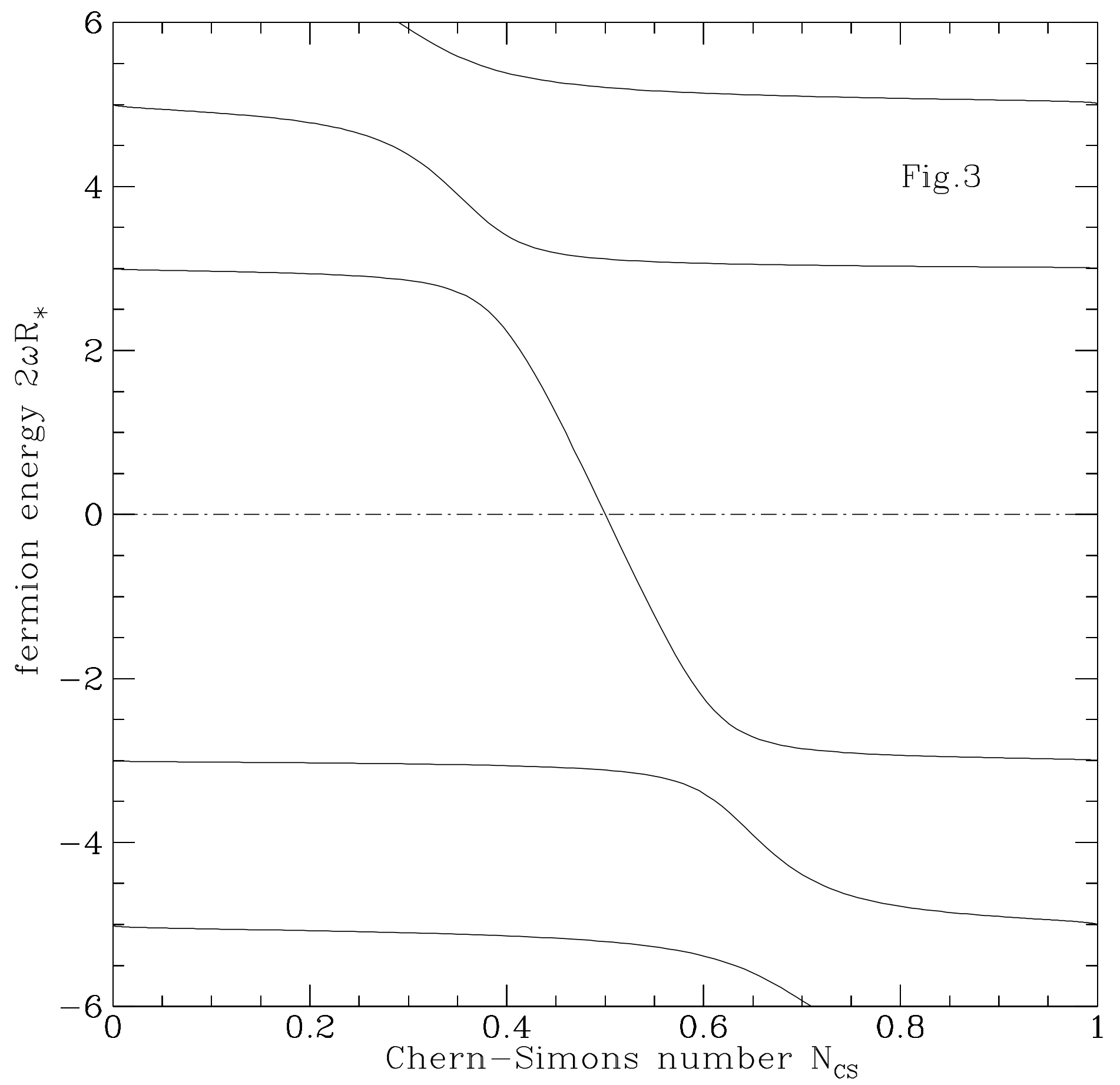

